name an isolated note without any referent is particularly refreshing and insightful. This phenomenon is one aspect of the psychology of music that has garnered considerable public and scientific attention. Huron subtly questions the utility of absolute pitch, suggesting that we may have lost this ability over our evolutionary history because the adaptive pressures of our musical environments support listening to and producing music in terms of relative, rather than absolute, pitch.

Many such intellectual provocations take the reader on a fascinating journey into the inner workings of music and how it tickles the human mind.

Petr Janata is at the Center for Mind and Brain, University of California, Davis,

California 95616, USA.

\title{
Learning the mother tongue
}

\section{The Infinite Gift: How Children Learn and Unlearn the Languages of the World by Charles Yang \\ Scribner: 2006.288 pp. \$25}

\section{Annette Karmiloff-Smith}

Clearly nature and nurture both play vital roles in human development, and most scholars have now rejected the old 'either/or' controversy in favour of theories that invoke complex interactions between the two. However, the debate remains as to whether nature or nurture plays the greater role in shaping the developing brain's acquisition of a native tongue. This is partly because we lack testable theories of the precise processes by which genes and environment interact, and partly because many in the field have entrenched philosophical views about what it is to have human language. For some, consistent regularities in the physical and social environments to which children are exposed play a critical role; for others, the environment simply acts as a trigger for the functioning of our genetic endowment.

In The Infinite Gift, Charles Yang navigates between these two positions, endorsing without question Noam Chomsky's 'nativist' theory, which holds that the abstract human capacity for language is prespecified in our genes. But Yang gives the theory a new slant: he argues that children learn their mother tongue by unlearning all the other languages of the world. According to Yang, English children have a different language from adults, not because they occasionally speak imperfect English, but because they occasionally speak perfect Chinese (or Eskimo, French, German, Swahili...). He claims that childhood errors never violate the principles and parameters of the world's languages, and can be explained by translating them into other languages.

Yang argues that, during the early years, children engage in 'variational learning', whereby several languages simultaneously form part of the child's ongoing hypotheses. Gradually, through competition between grammars, the child hones in on the mother tongue: "every instance of language learning is just a bunch of parameters fighting for supremacy". Inspired by Darwin and using some compelling examples, Yang asserts that language learning and historical change can both be explained by the mechanism of natural selection. Yang's nativist approach seems more dynamic than that of many of Chomsky's disciples, placing more emphasis on learning by the child.

Engagingly written, with fascinating examples conveying the author's enthusiasm for his topic, Yang's book vacillates between targeting parents, students and academic peers. He doesn't mention competing theories, so the naive parent or student would be forgiven for thinking that the whole scientific community concurs on how language is acquired. Yet a growing body of cross-linguistic work based on construction grammar has been emerging from evolutionary anthropology, offering the richest hypothesis-driven data sampling of early child language currently available. In my view, readers of the book would have benefited from being able to contrast chomskyan

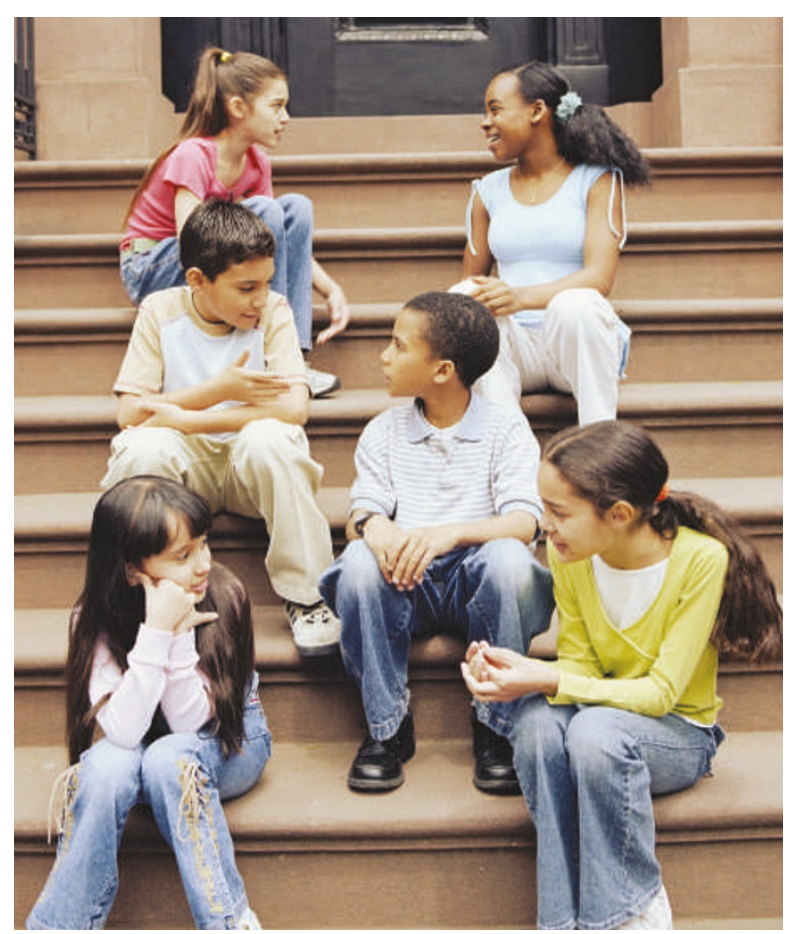

Speaking a different language: do children start with a grasp of all the world's languages before focusing on their mother tongue? theory with at least one other. Instead, we are presented with supporting experimental work emanating solely from Chomsky's disciples. For instance, Yang mentions work on a genetic disorder called Williams syndrome, claiming that moderate to severe mental retardation coexists with unimpaired linguistic ability. But he fails to bring to the reader's attention the work of numerous labs demonstrating that the general intelligence of those with Williams syndrome is actually in the mild range, and that their linguistic ability is seriously delayed in childhood, follows an abnormal developmental trajectory, and reveals semantic, grammatical and pragmatic deficits in adolescence and adulthood.

To support his assertion that there are critical periods for language learning, Yang calls upon examples of feral children. Yet in her book Wild Boy (Sceptre, 2003), Jill Dawson made a convincing case regarding the 'wild boy of Aveyron' that it was not the lack of input at appropriate ages that impeded language acquisition, but the fact that the boy was severely autistic (perhaps also explaining why he was abandoned in the first place).

On the topic of genes, Yang claims that "the uniquely human ability for language must ultimately reside in some uniquely human genes". Yet elsewhere he recognizes that FOXP2, the so-called 'language gene', is also involved in other cognitive functions and the development of body parts. In fact, it is questionable whether FOXP2 is directly involved in language at all. Rather, it is indirectly involved in rapid speech perception and production, and is expressed in humans, apes, mice and birds. Despite the allelic difference of FOXP2 in humans, its function is similar across many species - the rapid coordination of intricately timed motor sequences. The gene is first expressed in many different cerebral regions, but over developmental time its expression is increasingly confined to the cerebellum.

Let me conclude by drawing attention to Yang's justification of his allegiance to a nativist theory; as he says, "we cannot poke around the child's brain". Such an assertion is rather outdated, as we can now examine online processing in infant brains by using high-density evoked related potentials, near-infrared spectroscopy, and other non-invasive methods. These have already shown that the infant cortex starts out highly interconnected, with widespread activity occurring for different inputs. Only with time does the infant brain become specialized and localized for different functions. Such studies are likely to open exciting windows on how the developing brain acquires language.

Annette Karmiloff-Smith is head of the Neurocognitive Development Unit, Institute of Child Health, 30 Guilford Street, London WC1N 1EH, UK. 